\title{
Microbes, Transmission Routes and Survival Outside the Body
}

\begin{abstract}
Microbes like bacteria, virus, parasites and fungi may naturally colonize skin and mucous membranes without any sign of illness, for a longer or shorter period, in all humans, animals, fish, parasites, plants and all other living beings. Some types may be more invasive in human tissue than others. Many microbes are freeliving in the environment - in water, soil and air and on equipment — as a part of the normal microbial flora on the Earth. Most of them are not dangerous and live in peaceful symbiosis with other living beings and may also be transferred between living species, from man to animal or man to plants and environmentand vice versa. New and old human pathogenic microbes are increasing all over the world. Some agents, like drug-resistant bacteria and highly pathogenic viruses, are more dangerous than others, and some microbes may cause chronic devastating diseases. Transmission routes depend on the robustness of the microbe in the environment, virulence, infectious dose, anatomical site in the body, etc. Pathogenic microbes are spread by contact, air, water, food, beverages, contaminated equipment and environment and are more seldom vector-borne, by insects or animals. The following chapter is focused on the most frequent pathogenic microbes, their preselected localization in the body, transmission routes and survival in the environment.
\end{abstract}

\section{Keywords}

Microbes · Pathogenic microbes · Virulence $\cdot$ Predilection · Biological material · Anatomical site $\cdot$ Environment $\cdot$ Survival $\cdot$ Transmission $\cdot$ Spread of infection

\subsection{Microbes are Mostly Normal, Nonpathogenic Flora}

Microbes like bacteria, virus, parasites and fungi may naturally colonize skin and mucous membranes without any sign of illness, for a longer or shorter period, in all humans, animals, parasites, plants and all other living being [1-6]. Some types may 
be more invasive in human tissue than others, partly via mucous membranes and lesions in the skin.

Many microbes are free-living in the environment - in water, soil and air and on equipment - as a part of the normal microbial flora on the Earth. Most of them are not dangerous and live in peaceful symbiosis with other living beings and may also be transferred between living species, from man to animal or man to plants and environment-and vice versa [1-6]. Microbes, like bacteria, may outnumber us by a factor of $10^{22}$, are heavier than us by a factor of $10^{8}$, have existed on Earth more than 1000 times longer than us and may undergo 500,000 generations in just one of our generations [7].

The existence of humans and animals is dependent on a rich and active bacterial flora in the gut, participating in the decomposition of food substances to energy and growth. Large amounts of bacteria in the gut (1-2 kg) and on the skin is a normal condition. Humans are releasing microbes into the environment and air wherever they move and-at the same time-are picking up new microbes from the environment.

\subsection{Pathogenic Microbes: New and Old}

Pathogenic microbes cause illness in most humans if introduced into sterile tissue. "Opportunistic" microbes may cause problems in people with reduced immune defence and/or if large amounts are introduced in sterile tissue. Nonpathogenic microbes nearly never cause illness in humans [1-3]. Human pathogenic microbes often survive for a long time outside the body-in the environment [1-6]. Therefore, they are special threats to patients, personnel and visitors in healthcare institutions where there often is an accumulation of infectious diseases.

New and old human pathogenic microbes are increasing all over the world. Some agents, like drug-resistant bacteria (methicillin-resistant Staphylococcus aureus, MRSA, multidrug-resistant tubercle bacilli and others) and highly pathogenic viruses (Ebola, SARS and others), are more dangerous than others. Other viral agents may cause chronic devastating diseases like HIV and hepatitis B and C.

Microbes may have preselected locations and tissues in the host, like influenza virus, pneumococci and tuberculosis mostly in lungs, hepatitis viruses in the liver and blood, Clostridium difficile and norovirus in the gut, coagulase negative staphylococci on the skin, etc.

\subsection{Transmission Routes: Spread of Infection}

The transmission routes of microbes are many and different and depend on the robustness of the microbe in the environment, climate and temperature, virulence, infectious dose, etc. Pathogenic microbes are spread by contact, air, water, food, beverages, contaminated equipment and environment and are more seldom vectorborne, by insects or animals. Drug-resistant microbes and/or resistance genes are 
common on the global food market for humans, animals and fish [8-11]. In addition, increased mobility, climatic changes, overcrowding, war and disasters, poor hygiene and poor infection control are increasing the transmission rate. Prudent use of antimicrobial drugs, proper hygiene and good infection control for humans, animals and fish are essential for stopping spread of infections [12-19].

\section{Microbes: pathways and survival outside the body-in environment}

\begin{tabular}{|c|c|c|}
\hline Biological material-location/microbes & $\begin{array}{l}\text { Transmission } \\
\text { routes }\end{array}$ & $\begin{array}{l}\text { Lifetime in the } \\
\text { environment }^{1}\end{array}$ \\
\hline \multicolumn{3}{|l|}{ Wound } \\
\hline Staphylococcus aureus & C/A [2] & 3-10 months \\
\hline Methicillin-resistant S. aureus (MRSA) & $\mathrm{C} / \mathrm{A}$ & $3-10$ months \\
\hline $\begin{array}{l}\text { Gram-negative rods (Escherichia coli, Klebsiella, } \\
\text { Enterobacter, Serratia, Proteus, Pseudomonas, } \\
\text { Acinetobacter, etc.) }\end{array}$ & $\mathrm{C} /(\mathrm{A})$ & 2 days -16 months \\
\hline Multidrug-resistant gram-negative rods & C/A & 2 days -16 months \\
\hline \multicolumn{3}{|l|}{$\begin{array}{l}\text { - ESBL: E. coli, Enterobacter, Citrobacter, } \\
\text { Klebsiella, Serratia, etc. }\end{array}$} \\
\hline $\begin{array}{l}\text { - Others: Pseudomonas, Acinetobacter, } \\
\text { Stenotrophomonas maltophilia } \\
\text { - New multidrug-resistant with NDM-1 gene; } \\
\text { resistant to carbapenem and most other } \\
\text { antibiotics }\end{array}$ & & Klebsiella $>2.5$ years \\
\hline Enterococci, including VRE & $\mathrm{C}$ & 2 months -4 years \\
\hline Groups A, B, C, G streptococci & $\mathrm{C}$ & $1-7$ months \\
\hline \multicolumn{3}{|l|}{ Prosthesis, foreign body } \\
\hline Coagulase-negative staphylococci & C/A & 3 months \\
\hline S. aureus and MRSA & $\mathrm{C} / \mathrm{A}$ & $3-10$ months \\
\hline Gram-negative rods & $\mathrm{C}$ & 2 days -16 months \\
\hline Enterococci, including VRE & $\mathrm{C}$ & 2 months -4 years \\
\hline Candida & $\mathrm{C}$ & Several months \\
\hline \multicolumn{3}{|l|}{ Blood and tissue fluids } \\
\hline Hepatitis A, B, C, D, E & $\mathrm{B}(\mathrm{C})[3]$ & 3 days-year \\
\hline HIV & $\mathrm{B}(\mathrm{C})[3]$ & 3-14 days \\
\hline Other retroviruses (HTLV-I, HTLV-II) & B & Days \\
\hline Parvovirus B19 and others & B & $>1$ year \\
\hline Cytomegalovirus & $\mathrm{B} / \mathrm{C}$ & Hours to days \\
\hline Prions & $\mathrm{B}^{4}$ & Infinite? \\
\hline Malaria and other blood parasites & B, insects & Varies \\
\hline Yellow fever virus & B, mosquito & Varies \\
\hline Haemorrhagic fever virus & $\mathrm{B} / \mathrm{C} / \mathrm{A}$, insects & Varies-long? \\
\hline \multicolumn{3}{|l|}{ Respiratory tract infections } \\
\hline Pneumococci, including penicillin resistant & $\mathrm{C} / \mathrm{A}$ & Hours-20 d \\
\hline $\begin{array}{l}\text { Gram-negative rod bacteria ( } E \text { coli, Klebsiella, } \\
\text { Enterobacter, Acinetobacter, Serratia, } \\
\text { Pseudomonas, Morganella, etc.) }\end{array}$ & $\mathrm{C} / \mathrm{A}$ & 2 days -16 months \\
\hline Haemophilus influenzae & $\mathrm{C} / \mathrm{A}$ & Hours-12 days \\
\hline S. aureus & $\mathrm{C} / \mathrm{A}$ & 3-10 months \\
\hline
\end{tabular}




\begin{tabular}{|c|c|c|}
\hline Biological material-location/microbes & $\begin{array}{l}\text { Transmission } \\
\text { routes }\end{array}$ & $\begin{array}{l}\text { Lifetime in the } \\
\text { environment }^{1}\end{array}$ \\
\hline $\begin{array}{l}\text { Chlamydia pneumoniae/Mycoplasma } \\
\text { pneumoniae }\end{array}$ & C/A L & Hours-days \\
\hline Legionella pneumophila, etc. & A/C/water & Water: infinity \\
\hline Mycobacterium tuberculosis (tuberculosis) & C/A & 1 year \\
\hline Neisseria meningitidis; meningococcus & $\mathrm{C} / \mathrm{A}$ & Hours \\
\hline Pertussis; Bordetella pertussis & $\mathrm{C} / \mathrm{A}$ & $3-5$ days \\
\hline $\begin{array}{l}\text { Influenza viruses A, B, parainfluenza, adeno, } \\
\text { rhino, entero, corona }\end{array}$ & $\mathrm{C} / \mathrm{A}$ & Hours-20 d \\
\hline RSV (respiratory syncytial virus) & $\mathrm{C} / \mathrm{A}$ & Hours $-3 \mathrm{~d}$ \\
\hline Metapneumovirus & $\mathrm{C} / \mathrm{A}$ & Unknown \\
\hline $\begin{array}{l}\text { Coronavirus OC43 (cold virus) } \\
\text { Human bocavirus (Parvoviridae) }\end{array}$ & $\mathrm{C} / \mathrm{A}$ & Unknown \\
\hline Avian influenza & $\mathrm{A} / \mathrm{C} / \mathrm{B}$ & Days to 6 weeks \\
\hline SARS, MERS & $\mathrm{A} / \mathrm{C} / \mathrm{B}$ & 3-4 weeks \\
\hline Haemorrhagic fever virus & $\mathrm{A} / \mathrm{C} / \mathrm{B}$ & Varies prolong \\
\hline \multicolumn{3}{|l|}{ Faeces/gastrointestinal tract } \\
\hline $\begin{array}{l}\text { Intestinal pathogenic bacteria (Salmonella, } \\
\text { Shigella, Campylobacter, Yersinia, } \\
\text { Enteropathogenic E. coli, Helicobacter, Vibrio } \\
\text { cholerae) }\end{array}$ & C/food/water & 4-14 days -4.2 years \\
\hline Clostridium difficile (spores) & $\mathrm{C}$ & Years \\
\hline Listeria monocytogenes & C/food/water & Long, chilled \\
\hline $\begin{array}{l}\text { Bacterial toxins (E coli, }, \text {. aureus, Clostridium } \\
\text { perfringens, Salmonella, etc.) }\end{array}$ & C/food & Hours to days \\
\hline Rota/adeno/norovirus (Norwalk) & C/food/water & 60-90 days \\
\hline Hepatitis A, hepatitis E & C/food/water & 14 days -2 months \\
\hline SARS, MERS and avian influenza virus & $\mathrm{C} / \mathrm{A} / \mathrm{B}$ & $3-6$ weeks \\
\hline $\begin{array}{l}\text { Parasites } \\
\text { Giardia lamblia, Cryptosporidium parvum, } \\
\text { Entamoeba histolytica, schistosomiasis, Ascaris, } \\
\text { hookworm, trichuriasis, strongyloidiasis, etc. }\end{array}$ & C/water/food & Weeks-months-year \\
\hline \multicolumn{3}{|l|}{ Urinary tract infections } \\
\hline E. coli & $\mathrm{C}$ & Hours-16 months \\
\hline $\begin{array}{l}\text { Other gram-negative rods (Proteus, Klebsiella, } \\
\text { Citrobacter, Enterobacter, Pseudomonas, etc.) }\end{array}$ & $\mathrm{C}$ & Hours to 16 months \\
\hline Enterococci & $\mathrm{C}$ & $2 \mathrm{mndr}-4$ years \\
\hline $\begin{array}{l}\text { Resistant bacteria (VRE, ESBL, CRE, MRSA, } \\
\text { etc.) }\end{array}$ & $\mathrm{C} / \mathrm{A}$ & days -4 years \\
\hline \multicolumn{3}{|l|}{ Others } \\
\hline $\begin{array}{l}\text { Borrelia burgdorferi, Ehrlichia sp. and less } \\
\text { frequent bacteria }\end{array}$ & $\begin{array}{l}\text { Ticks/insects, } \\
\text { etc. }\end{array}$ & Varies \\
\hline Varicella zoster virus & $\mathrm{A} / \mathrm{C}$ & Hours-days \\
\hline Herpes simplex virus & $\mathrm{C}$ & Hours-2 months \\
\hline (Measles, rubella, parotitis virus) & $\mathrm{A} / \mathrm{C}$ & Hours-days \\
\hline \multicolumn{3}{|l|}{ Highly pathogenic microbes } \\
\hline Multidrug-resistant Mycobacterium tuberculosis & $\mathrm{A} / \mathrm{C}$ & 1 year \\
\hline
\end{tabular}




\begin{tabular}{l|l|l}
\hline Biological material-location/microbes & $\begin{array}{l}\text { Transmission } \\
\text { routes }\end{array}$ & $\begin{array}{l}\text { Lifetime in the } \\
\text { environment }^{1}\end{array}$ \\
\hline SARS, MERS virus & A/C/B & $2-6$ weeks ++ \\
\hline Haemorrhagic fever virus & A/C/B & $2-3$ weeks \\
\hline (Lassa, Ebola, Sabia, Marburg virus, etc.) & A/C/B & Weeks \\
\hline Avian influenza & A/C/B & Days-6 weeks ++ \\
\hline
\end{tabular}

From Refs. [1-6]

1. Lifetime in the environment, outside the body. Most gram-negative rod bacteria live almost infinite in water; some also multiply rapidly in water (Klebsiella, Enterobacter, Pseudomonas, Proteus, etc.).

2. $\mathrm{C}=$ contact transmission, $\mathrm{A}=$ airborne transmission (including droplets), $\mathrm{B}=$ blood-borne infection, $\mathrm{v}=$ variable, $\mathrm{k}=$ short life outside the body, ++ can be long life in optimal environment.

3. May be transmitted by mucous membrane contact, especially lesions of the mucosa.

4. Infectious protein molecules; spongiform encephalopathy.

\section{References}

1. Mandell, Douglas, Bennet's, editors. Principles and practice of infectious diseases. 8th ed. Philadelphia: Churchill Livingston; 2015.

2. Andersen BM. Bacteria and disease. Oslo: Gyldendal academic; 2005.

3. Andersen BM. Handbook in hygiene and infection control for hospitals. Part 1. Microbiology and infection control. Fagbokforlaget; 2014.

4. Kramer A, Schebke I, Kampf G. How long do nosocomial pathogens persist on the surfaces? A systematic review. BMC Infect Dis. 2006;6:130-8.

5. Boyce JM. Environmental contamination makes an important contribution to hospital infection. J Hosp Infect. 2007;65:50-4.

6. Otter JA, French GL. Survival of nosocomial bacteria and spores on surfaces and inactivation by hydrogen peroxide vapour (HPV). J Clin Microbiol. 2009;47:205-7.

7. The Board of Global Health and Institute of Medicine. Ending the war metaphor: the changing agenda for un-ravelling the host-microbe relationship - workshop summary. Washington (DC): The National Academies Press; 2006.

8. Andersen BM. Food infection is a neglected problem. Aftenposten; 2014.

9. Paterson GK, Larsen J, Harrison EM, et al. First detection of livestock-associated methicillinresistant Staphylococcus aureus CC398 in bulk tank milk in the United Kingdom, January to July 2012. Eur Secur. 2012;17:20337.

10. Casey JA, Curriero FC, Cosgrove SE, Nachman KE, Schwartz BS. High-density livestock operations. Crop field application of manure, and risk of community-associated methicillinresistant Staphylococcus aureus infection in Pennsylvania. JAMA Intern Med. 2013.

11. ECDC Surveillance Reports. Summary of the latest data on antibiotic resistance in Europe; 2017.

12. Dancer SJ, Kirkpatrick P, Corcoran DS, Christison F, Farmer D, Robertson C. Approaching zero: temporal effect of a restrictive antibiotic policy on hospital-acquired Clostridium difficile, extended-spectrum beta-lactamase- producing coliforms and methicillin-resistant Staphylococcus aureus. Internat J Antimicrob Agents. 2013;41:137-42.

13. NORM/NORM-VET 2013-2017. Usage of antimicrobial agents and occurrence of antimicrobial resistance in Norway. Troms $\varnothing / O s l o ; 2013$.

14. Ramirez E, Gomez-Gil R, Borobia AM, et al. Improving linezolid use decreases the incidence of resistance among gram-positive microorganisms. Internat J Antimicrob Agents. 2013;41:174-8. 
15. Galar A, Leiva J, Espinosa M, Guillen-Grima F, Hernaez S, Yuste JR. Clinical and economic evaluation of the impact of rapid microbiological diagnostic testing. J Infect. 2012;65:302-9.

16. Sexton T, Clarke P, O'Neill E, Dillane T, Humphreys H. Environmental reservoirs of methicillin-resistant Staphylococcus aureus in isolation rooms: correlation with patient isolates and implications of hospital hygiene. J Hosp Infect. 2006;62:187-94.

17. Dancer SJ. Mopping up hospital infection. J Hosp Infect. 1999;43:85-100.

18. Dancer SJ. The role of environmental cleaning in the control of hospital-acquired infection. $J$ Hosp Infect. 2009;73:378-85.

19. Andersen BM, Rasch M, Kvist J, Tollefsen T, Lukkassen R, Sandvik L, Welo A. Floor cleaning: effect on bacteria and organic materials in hospital rooms. J Hosp Infect. 2009;71:57-65. 